populations, validity of biomonitoring methods etc. The performance of the various exposure assessment methods will be compared and contrasted within existing epidemiological studies.

Results The main outcome will be the validation of widely accepted and easily adaptable semi-quantitative individual-based exposure assessment methods against measured levels of urinary PPP metabolites in a broad range of settings. Additionally, the study will compare the reliability and performance of several grouped- and individual-based exposure assessment methods in the frame of existing epidemiological studies.

Conclusion The project is due to commence in September 2017. We propose to present the study protocols, rationale and outcomes of a mini-symposium to be held at EPICOH 2017.

(C) British Crown copyright (2017). This work is funded by the European Crop Protection Association (ECPA). The Health and Safety Laboratory is the commercial arm of the Health and Safety Executive (HSE); the paper's contents including any opinions and/or conclusions expressed, are those of the authors alone and do not necessarily reflect ECPA or HSE policy.

\section{MEASURING AIRBORNE EXPOSURE OF FRENCH FARMERS DURING WORK RELATED TO LIVESTOCK AND TO HARVESTING OF VARIOUS CROPS: THE AIREXPA PROJECT}

\begin{abstract}
${ }^{1,2,3} \mathrm{M}$ Boulanger, ${ }^{4} \mathrm{~V}$ Bouchart, ${ }^{1,5} \mathrm{Y}$ Lecluse, ${ }^{1,5} \mathrm{R}$ Pons, ${ }^{1,2,3} \mathrm{~B}$ Clin, ${ }^{6,7} \mid \mathrm{Baldi},{ }^{1,2,5} \mathrm{P}$ Lebailly, ${ }^{1,5}$ S Tual. ${ }^{1}$ INSERM, UMR 1086 Cancers et Préventions, Caen, France; ${ }^{2}$ Université de Caen Normandie, Caen, France; ${ }^{3} \mathrm{CHU}$ de Caen, Service de Pathologie Professionnelle, Caen, France; ${ }^{4}$ Laboratoire LABEO Frank Duncombe, Caen, France; ${ }^{5}$ Centre de Lutte Contre le Cancer François Baclesse, Caen, France; ${ }^{6}$ Université de Bordeaux, Inserm, team EPICENE, Bordeaux, France; ${ }^{7} \mathrm{CHU}$ de Bordeaux, Service de Médecine du Travail et Pathologies professionnelles, Bordeaux, France
\end{abstract}

\subsection{6/oemed-2018-ICOHabstracts. 1322}

Introduction Beyond pesticide exposure, farmers are likely to be exposed to several air pollutants. Some of them are of particular interest, either because of their known carcinogenic properties, for lung cancer (total dust, Diesel exhaust, crystalline silica,) or other cancer sites (mycotoxins) or because of a supposed protective effect on lung cancer risk (endotoxins). However, except for endotoxins, very few studies, assessed farmers' exposure levels by individual samplings. Our study aims at describing the levels, and assessing the determinants, of agricultural exposure to these air pollutants.

Methods We assessed French farmers' individual exposure to several air contaminants: (1) inhalable endotoxins and mycotoxins during various tasks in several breedings (cattle, horse) and 3 crops (grassland, wheat/barley, peas); (2) respirable crystalline silica during harvesting of these 3 crops; (3) respirable elementary carbon during the use of Dieselized farm equipment. Inhalable dusts, up to $20 \mu$, were assessed in real time.

Sampling was performed during the whole activity, and endotoxin exposure was also assessed for each task separately. Field monitors followed the farmers and collected detailed information on the activity.
Results The field study is on-going, we already observed around 40 individual measurements in 20 different farms. The protocol was judged acceptable by the participants. Sampling duration ranges from 80 to $240 \mathrm{~min}$, depending on the type of activity. First results from real-time dust measurements suggest a higher exposure during stables cleaning and mulching (mean concentration of inhalable dusts: $0.545 \mathrm{mg} / \mathrm{m}^{3}$ ). A total of several hundreds of measurements is expected in various types of farms and working conditions (number of animals, cultivated area, type of equipment).

Discussion We hope our study will improve the assessment of occupational health hazards in agriculture, and thus primary prevention, by quantifying the levels of individual exposure to several air pollutants.

\section{IS PLASMA CHOLINESTERASE A RELIABLE BIOMARKER IN LONG-TERM EXPOSURE TO CHOLINESTERASE INHIBITORS?}

${ }^{1}$ Anaclaudia G Fassa*, ${ }^{1}$ Neice Muller X Faria, ${ }^{2}$ Rodrigo D Meucci, ${ }^{1}$ Nadia S Fiori, ${ }^{1}$ Maria Laura V Carret, ${ }^{3}$ Carlos Augusto Mello Silva. 'Pelotas Federal University (UFPEL), Pelotas, Rio Grande do Sul, Brazil; ${ }^{2}$ Federal University of Rio Grande FURG, Rio Grande, Rio Grande do Sul, Brazil; ${ }^{3}$ Toxicology Information Centre of Rio Grande do Sul CIT-RS, Porto Alegre, Rio Grande do Sul, Brazil

\subsection{6/oemed-2018-ICOHabstracts. 1323}

Introduction The use of insecticides has been intensified in Brazilian agricultural production and control of endemic diseases. Plasma - or Butiryl -Cholinesterase (BCHE) is used for biological monitoring of organophosphates (OP) and carbamate exposure. Despite legal requirements, there are controversies about this monitoring.

Methods Cross - sectional study carried out among pesticide applicators in tobacco farming. Sociodemographic, occupational data, PPE use, pesticide- related-symptoms (PRS) and standardised medical examination were collected BCHE was measure at low and high exposure period. Pesticide exposure was characterised by using chemical types and days from last exposure. The assessment by physicians and toxicologists classified poisoning by pesticides as possible or probable. The analysis was made by chi-square test, $t$ test for comparison of two means and Poisson regression.

Results Out of 492 smokers studied, 43\% had recent exposure to $\mathrm{OP}$ or Carbamates, with average $\mathrm{BCHE}$ increase in intensive period $(\mathrm{p}<0.001)$. Five people $(2.4 \%)$ presented a reduction of $\geq 20 \%$ in BCHE. There was more BCHE reduction in the most exposed group. There were no association with any pesticide poisoning criteria, BCHE levels or use of PPE.

Conclusions Although consistent with some studies, the findings of BCHE contrast with other authors. Possible explanations include tolerance to long term exposure, differences in analytical methodology, genetic polymorphism, other diseases and limitations with the gold standard. Future studies should investigate these results to define better recommendations for occupational monitoring, including periodicity and cutoff points. New biomarkers should be developed to exposure monitoring of cholinesterase inhibitors and other pesticides. 